\title{
Intracranial pressure directly predicts headache morbidity in idiopathic intracranial hypertension
}

S. P. Mollan', B. R. Wakerley ${ }^{2,3}$, Z. Alimajstorovic ${ }^{2}$, J. Mitchell ${ }^{2,3}$, R. Ottridge ${ }^{4}$, A. Yiangou ${ }^{2,3}$, M. Thaller ${ }^{2,3}$, A. Gupta ${ }^{4}$,

O. Grech ${ }^{2}$, G. Lavery ${ }^{2}$, K. Brock ${ }^{5}$ and A. J. Sinclair ${ }^{2,3^{*}}$

\begin{abstract}
Objective: Headache is the predominant disabler in idiopathic intracranial hypertension (IIH). The aim was to characterise headache and investigate the association with intracranial pressure.

Methods: IIH:WT was a randomised controlled parallel group multicentre trial in the United Kingdom investigating weight management methods in $\mathrm{IH}$. Participants with active $\mathrm{IH}$ (evidenced by papilloedema) and a body mass index $(\mathrm{BMI}) \geq 35 \mathrm{~kg} / \mathrm{m}^{2}$ were recruited. At baseline, 12 months and 24 months headache characteristics and quality of life outcome measures were collected and lumbar puncture measurements were performed.

Results: Sixty-six women with active $\| \mathrm{H}$ were included with a mean age of 32.0 years (SD \pm 7.8 ), and mean body mass index of $43.9 \pm 7.0 \mathrm{~kg} / \mathrm{m}^{2}$. The headache phenotype was migraine-like in $90 \%$. Headache severity correlated with ICP at baseline $(r=0.285 ; p=0.024)$; change in headache severity and monthly headache days correlated with change in ICP at 12 months ( $r=0.454, p=0.001$ and $r=0.419, p=0.002$ respectively). Cutaneous allodynia was significantly correlated with ICP at 12 months. ( $r=0.479, p<0.001)$. Boot strap analysis noted a positive association between ICP at 12 and 24 months and enabled prediction of both change in headache severity and monthly headache days. ICP was associated with significant improvements in quality of life (SF-36).

Conclusions: We demonstrate a positive relationship between ICP and headache and cutaneous allodynia, which has not been previously reported in IH. Those with the greatest reduction in ICP over 12 months had the greatest reduction in headache frequency and severity; this was associated with improvement of quality of life measures.
\end{abstract}

Trial registration: This work provides Class lla evidence of the association of raised intracranial pressure and headache. ClinicalTrials.gov number, NCT02124486.

Keywords: Idiopathic intracranial hypertension, Migraine, Intracranial pressure, Allodynia, Calcitonin gene related peptide

\footnotetext{
* Correspondence: a.b.sinclair@bham.ac.uk

${ }^{2}$ University of Birmingham, Metabolic Neurology, Institute of Metabolism and Systems Research, Birmingham, UK

${ }^{3}$ Department of Neurology, University Hospitals Birmingham, Birmingham, UK

Full list of author information is available at the end of the article
}

\section{$\triangle B M C$}

(c) The Author(s). 2021 Open Access This article is licensed under a Creative Commons Attribution 4.0 International License, which permits use, sharing, adaptation, distribution and reproduction in any medium or format, as long as you give appropriate credit to the original author(s) and the source, provide a link to the Creative Commons licence, and indicate if changes were made. The images or other third party material in this article are included in the article's Creative Commons licence, unless indicated otherwise in a credit line to the material. If material is not included in the article's Creative Commons licence and your intended use is not permitted by statutory regulation or exceeds the permitted use, you will need to obtain permission directly from the copyright holder. To view a copy of this licence, visit http://creativecommons.org/licenses/by/4.0/ The Creative Commons Public Domain Dedication waiver (http://creativecommons.org/publicdomain/zero/1.0/) applies to the data made available in this article, unless otherwise stated in a credit line to the data. 


\section{Introduction}

Idiopathic intracranial hypertension (IIH) is a rare disease that is increasingly recognised $[1,2]$. It is characterized by raised intracranial pressure (ICP) in the absence of a structural cause on brain imaging [3, 4]. Although the exact cause of IIH remains unknown, disease development is associated with obesity and there is increasing evidence to suggest adipose dysfunction [5]. Headache is the predominant symptom and is prioritized highly by patients and physicians [6]. The headache in IIH remains under-characterized [7] and is associated with significant morbidity and reduced quality of life [8].

The International Classification of Headache Disorders (ICHD-3 beta) attributes headache in IIH to be associated with raised intracranial pressure and acknowledges that it often mimics primary headache disorders such as migraine [9]. While the International Headache Society stipulates the importance on relief of headache following removal of cerebrospinal fluid (CSF), evidence suggests this relief is far from universal [10] and can occur due to co-occurring headache disorders such as migraine [11]. Rarely some patients with evidence of raised ICP do not ever develop headache [12]. The pathogenesis of $\mathrm{IIH}$ headache remains unknown, as do the risk factors that propagate persistent headache. Calcitonin gene-related peptide (CGRP) monoclonal antibodies have been noted in a prospective study to significantly improve headache in patients with IIH and persistent post-IIH headache (resolved papilloedema), suggesting CGRP may modulate headache pain $[13,14]$.

The relationship between development of headache and raised ICP in IIH is poorly understood. A previous trial in 165 patients with newly diagnosed IIH [15] did not identify an association between intracranial pressure and headache. However, a question remains as to whether the role of ICP in driving ongoing headache in patients with chronic headache.

The aim of this analysis was to describe the headache characteristics in those with active IIH recruited to the multicentre IIH Weight Trial (IIH:WT), and to explore the relationship of headache features to ICP.

\section{Methods}

\section{Study procedures}

Between July 25, 2014 and May 25, 2017, 66 female patients were recruited to the multicentre randomised controlled trial IIH:WT, comparing the efficacy of a bariatric surgery pathway versus the dietary intervention Weight Watchers ${ }^{\mathrm{Tm}}$. All participants had active IIH with papilloedema and lumbar puncture opening pressures $\geq 25 \mathrm{~cm}$ cerebrospinal fluid, in accordance with agreed criteria for diagnosis of IIH [1]. The protocol and eligibility criteria have been previously published [16].
At baseline a detailed clinical, medication and standardized headache history was taken (including the location, character, associated symptoms, timing and exacerbating / relieving factors) by a physician with specialist training in headache phenotyping. Headache preventatives were permitted during the study, but any changes were recorded. As part of the trial anthropometric data including weight and height were recorded, and a lumbar puncture was performed. Headaches were characterized using ICHD-3beta criteria for primary and secondary headache disorders [9].

At baseline, 12 and 24 months all IIH patients were required to return a headache diary which included details of headache severity; headache duration; headache frequency (monthly headache days); and analgesic use (days per month). The headache severity was scored using a numerical rating scale (NRS) ranging from 0 (no pain) to 10 (the most severe pain level experienced by the subject). The NRS is favoured by patients and widely used in migraine trials [17].

Cutaneous allodynia symptoms were assessed during a headache using the patient-completed Allodynia Symptom Checklist-12 (ASC-12) in the week prior to their baseline and 12 month visit. Score ranges from $0=$ no symptoms to $24=$ severe symptoms; $0-2=$ no allodynia, 3-5 = mild allodynia, $6-8=$ moderate allodynia, 9 or more $=$ severe allodynia (supplemental methods). In addition, pressure allodynia was assessed in patients at baseline and at 12 months using three different weights of von Frey hairs (F1, $0.32 \mathrm{~g} ; \mathrm{F} 2,8.30 \mathrm{~g}$; and F3, 24 g) over V1-3 and C2-3 dermatomes bilaterally [18]. Patients rated pressure allodynia using a $100 \mathrm{~mm}$ visual analogue scale.

Outcome measures included the headache impact test-6 disability questionnaire (HIT-6); where little or no impact $=$ HIT -6 score $\leq 49 ;$ some impact $=$ HIT -6 score 50-55; substantial impact $=$ HIT-6 score 56-59; severe impact $=$ HIT -6 score $\geq 60$. Health-related quality of life was assessed using the Rand patient-reported 36Item Short Form Health Survey (SF-36). The eight sections of the SF-36 yielded two summary scores (physical component summary, PCS; and mental component summary, MCS).

\section{Statistical analysis}

Descriptive statistics were used to compare demographic characteristics. Analysis by trial arm was not part of the aims of the study. Statistical analysis was performed using GraphPad, version 8.3 (GraphPad Software, La Jolla, California, USA). Mean and standard deviations are provided for normally distributed variables, and median and range provided for nonnormally distributed variables. Pearson's correlation coefficient was computed where the variables were 
normally distributed and all assumptions were met, with Spearman's rank correlation used in other cases. Values were deemed statistically significant at $p<0.05$. Missing data, due to any absence or choice, were excluded from the analysis and not imputed.

Hierarchical regression models were generated, with data for all patients analysed in one model. Models contained population-level terms (i.e. terms that apply to each experimental unit) to reflect: 1) the mean baseline value (i.e. the intercept); 2) the mean change from baseline associated with each assessment time (i.e. time as a factor variable); 3 ) the extra mean change from baseline associated with each assessment time in the experimental arm (i.e. the interaction of treatment allocation and time as a factor variable). Additionally, hierarchical regression models contained random effects (i.e. terms that are specific to each experimental unit) to reflect the random deviations from the population-level mean value at baseline (i.e. random intercepts). For ICP, the random intercepts were estimated for each patient, with each of these parameters assumed to be exchangeable draws from a normal distribution.

To assess the relationship between ICP and headache, we have bootstrap resampling of the observed outcomes to generate alternative pairs of treatment effects. Alternative datasets were generated by resampling patients with replacement from the original treatment allocations to which they were randomised. The hierarchical regression model described above was fitted to each resampled dataset, producing a pair of treatment effects for the surrogate and clinical outcomes from a notional randomisation of patients. This process was repeated 1000 times. The resampled datasets within in each arm in each trial were the same size as the original datasets.

\section{Standard protocol approvals, registration, and patient consent}

The trial was approved by The National Research Ethics Committee West Midlands - The Black Country, on 28 February 2014 (14/WM/0011). All participants gave written consent after receiving detailed written information. The trial was registered, clinicaltrials.gov identifier: NCT02124486.

\section{Data availability statement}

Anonymised individual participant data will be made available along with the trial protocol and statistical analysis plan. Proposals should be made to the corresponding author and will be reviewed by the Birmingham Clinical Trials Unit Data Sharing Committee in discussion with the Chief Investigator. A formal Data Sharing Agreement may be required between respective organisations once release of the data is approved and before data can be released.

\section{Classification of evidence}

This work provides Class IIa evidence that headache is associated with intracranial pressure.

\section{Results}

\section{Patient characteristics}

Sixty-six persons (100\% women) with active IIH were included in the analysis (Table 1). Mean age at inclusion was 32.0 years ( $\mathrm{SD} \pm 7.8$, range $20-53$ years), and the mean body mass index was $43.9 \pm 7.0 \mathrm{~kg} / \mathrm{m}^{2}$ ranging from 35.3 to $63.3 \mathrm{~kg} / \mathrm{m}^{2}$. The median IIH disease duration was 1.1 years (IQR $0.5-2.6$ ) and ranging from 0.1 to 20.0 years. Forty-five participants $(68 \%)$ reported a previous history of migraine, of which 24 (53\%) reported onset in childhood (age $<18$ years).

Table 1 Characteristics of the study cohort at baseline

\begin{tabular}{ll}
\hline & $\begin{array}{l}\text { Total } \\
(\boldsymbol{n}=66)\end{array}$ \\
\hline Age in years, mean (SD) & $32(7.8)$ \\
Ethnicity, number (\%) & \\
$\quad$ White & $55(83)$ \\
$\quad$ Mixed & $5(8)$ \\
$\quad$ Asian & $1(1)$ \\
$\quad$ Black & $5(8)$ \\
Duration of IIH diagnosis, median (IQR) & $1.1(0.5-2.6)$ \\
Number on acetazolamide (\%) & $19(29)$ \\
Number on topiramate (\%) & $6(9)$ \\
Lumbar puncture opening pressure, cmCSF, mean (SD) & $35.5(7.0)$ \\
Weight, Kg (SD) & $118.5(21.1)$ \\
Body Mass Index (weight (kg)/ height (m²), mean (SD) & $43.9(7.0)$ \\
Smoking status - smoker & $n=27$ \\
Smoking intensity of 10 or more cigarettes per day, n (\%) & $18(67)$ \\
Family history of primary headache disorder, \% & $19(7 / 37)$ \\
Previous history of migraine, $\mathrm{n}$ (\%) & $45(68)$ \\
Duration of migraine, $\mathrm{n}$ (\%) & $n=45$ \\
Less than 1 year & $5(11)$ \\
1-5 years & $7(16)$ \\
5-10 years & $5(11)$ \\
10-20 years & $3(7)$ \\
More than 20 years & $1(2)$ \\
Since childhood (age < 18 years) & $24(53)$ \\
Headache preventative medication use, $\mathrm{n}(\%)$ & $n=18$ \\
\hline
\end{tabular}




\section{Headache characteristics at baseline}

Within this cohort 65 participants (98\%) reported headache at the time of their IIH diagnosis. At the time of the baseline assessment 63 (95\%) reported headache. In some cases patients described more than one type of headache. The headache phenotype was migraine-like in 57 of 63 (90\%) and of those, 23 (40\%) described migraine aura. Of those with migraine-like headaches 40 (70\%) had a phenotype consistent with chronic migraine-like headaches $(>15$ headache days per month of which $>8$ are migraine-like), while 17 (30\%) had episodic migraine-like headaches (less than 15 headache days per month) [8]. At the time of study assessment, 44 (67\%) had headache, which fulfilled the diagnostic criteria for headaches attributed to IIH. 23 (35\%) patients fulfilled the criteria for medication-overuse headaches. There was one patient who had headaches not attributable to either migraine-like or attributable to $\mathrm{IIH}$, and had tension-type headache.

Headache location was predominately bilateral 76\%; with fewer reporting unilateral pain, $20 \%$ being on the right side and $18 \%$ being on the left side (note these were not mutually exclusive responses, Supplemental Table 1). Headache pain was typically throbbing $73 \%$, but also a pressure sensation $55 \%$ and less commonly stabbing $11 \%$ or shooting $7 \%$. Photophobia and phonophobia were described in $81 \%$ and $60 \%$, respectively, with fewer (19\%) describing osmophobia. 70\% described nausea and $18 \%$ experienced vomiting with their headache attacks. Dizziness was reported in 33\%. Headaches on waking were reported in $12 \%$. Autonomic features were rarely reported $5 \%$. Pulsatile tinnitus was a feature in $74 \%$. Headaches were exacerbated by physical activity in $53 \%$; by lying flat in $32 \%$; on bending $31 \%$; and on Valsalva manoeuvre in $23 \%$. (Table 3).

\section{Headache burden}

Headache disability, as measured by the Headache Impact Test (HIT-6) questionnaire, had a mean score of 65 (SD \pm 7.3 ) at baseline. Mean headache severity was 5.0 (SD \pm 2.0$)$ and mean daily duration of headache was 8.2 h (SD \pm 6.3 ). Monthly headache days were mean 22.2 days (SD \pm 7.8 ) and mean monthly analgesic use was 12.3 days (9.0).

By 12 months $76 \%$ of participants reported ongoing headache. The mean headache severity had improved (3.6 ( $\mathrm{SD} \pm 2.9$ ), with a concurrent reduction in the headache frequency (monthly headache days $15.1(\mathrm{SD} \pm 11.5)$ ) and monthly analgesic use (8.6 (SD \pm 9.8$))$. No parameter reached statistical significance.

\section{Medications at baseline}

At baseline 38\% participants were receiving medication specifically to lower ICP. This included $29 \%$ receiving
Acetazolamide, and 9\% Topiramate. 5\% of participants were receiving diuretics (Bendroflumethiazide, $n=1$; Furosemide, $n=1$; and Co-amilofruse, $n=1$ ). $27 \%$ of participants were receiving medication for prevention of headache (migraine and/or tension-type headache) (beta blocker, $n=1$; tricyclic antidepressant, $n=7$; anticonvulsant, $n=8$; other, $n=2$ ) (Table 2).

\section{Relationship of ICP to headache}

The whole cohort mean lumbar puncture opening pressure (LP OP) at baseline was $34.7 \mathrm{cmCSF}$ (SD 5.7) which reduced to 28.95 (SD 7.7) and 26.8 (SD $8.0)$ by 12 and 24 months respectively. Headache severity at baseline correlated with ICP (Fig. 1a, $r=$ $0.285, p=0.024)$ although the monthly headache days did not. The reduction in ICP over 12 months correlated with the reduction in headache severity and monthly headache days (MHD) (Fig. 1b and c, $r=$ $0.454, p=0.001$ and $r=0.419, p=0.002$ respectively). This relationship continued at 24 months but did not reach statistical significance.

Table 2 Outcome measures, headache phenotype and ictal cutaneous allodynia at baseline

\begin{tabular}{ll}
\hline Headache at baseline, $\mathrm{n}(\%)$ & $63(95 \%)$ \\
Headache severity, verbal rating scale (0-10), mean (SD) & $5.0(2.0)$ \\
Headache duration (hours), mean (SD) & $8.2(6.3)$ \\
Headache frequency (days per month), mean (SD) & $22.2(7.8)$ \\
Analgesic use (days per month), mean (SD) & $12.3(9.0)$ \\
HIT-6 score, mean (SD) & $65(7.3)$ \\
SF-36, physical component score, mean (SD) $n=60$ & $28.7(12.7)$ \\
SF-36, mental component score, mean (SD) $n=60$ & $37.7(11.0)$ \\
Headache phenotypes (not mutually exclusive), $n$ (\%) & $n=66$ \\
No headache & $1(2 \%)$ \\
Migraine-like & $57(86 \%)$ \\
Migraine-like without aura & $34(60 \%)$ \\
Migraine-like with aura & $23(40 \%)$ \\
Chronic migraine-like & $40(70 \%)$ \\
Episodic migraine-like & $17(30 \%)$ \\
Headache attributed to IIH & $44(67 \%)$ \\
Medication-overuse & $23(35 \%)$ \\
Tension-like & $1(2 \%)$ \\
Cutaneous allodynia (ictal), mean (SD), $n=58$ & $19.16(5.79)$ \\
None (0-2) & $0(0 \%)$ \\
Mild (3-5) & $0(0 \%)$ \\
Moderate (6-8) & $2(3.4 \%)$ \\
Severe (9+) & $56(96.6 \%)$ \\
\hline
\end{tabular}



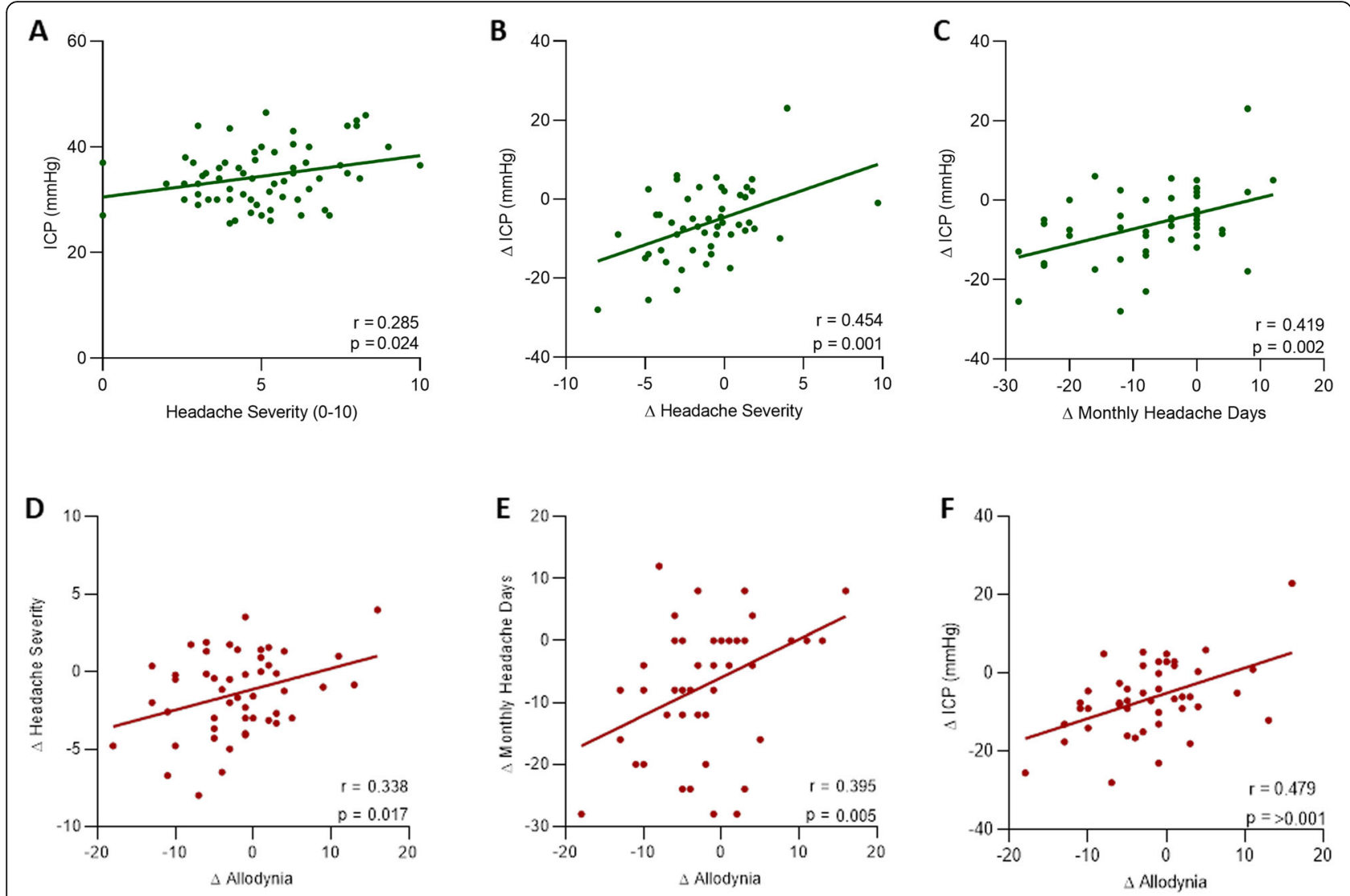

Fig. 1 Relationship between ICP and headache. Correlation of baseline headache severity (HS) against baseline intracranial pressure (ICP) (A); ICP correlated with change in HS (B) change in monthly headache days (MHD) (C) Change in MHD correlated with ICP between baseline and 12 months. Correlation of the change allodynia scores between baseline and 12 months against change in HS (D); change in MHD (E); ICP (F) between baseline and 12 months

\section{Relationship between cutaneous allodynia and ICP}

At baseline 58/63 (92\%) reported ictal cutaneous allodynia with a mean cutaneous allodynia score of 19.2 $(\mathrm{SD} \pm 5.79)$ and reducing at 12 months to a mean of 17.1 (SD \pm 7.20$)(p=0.01)$. There was no relationship between the allodynia score and headache severity and MHD at baseline. By 12 month MHD correlated with the allodynia score $(r=0.331, p=0.015)$. The change in MHD and headache severity over 12 months correlated with the improving allodynia score (Fig. 1d and e, $r=$ $0.395, p=0.005$ and $r=0.338, p=0.017$ respectively). The change in allodynia over 12 months also correlated with the change in ICP (Fig. 1e, $r=0.479, p<0.001$ ). There was no relationship between body mass index (BMI) and allodynia. Pressure allodynia did not change significantly between baseline and 12 months and was not associated with headache severity, MHD or ICP (Supplemental Table 2).

\section{Predicting the effects of ICP on headache outcomes}

Analysis identified a positive association between ICP and headache severity and MHD at 12 and 24 months, with a larger change in intracranial pressure coinciding with a larger change in headache measures (Fig. 2a). The bootstrapped analysis data points (Fig. 2) represent trial outcomes, each as likely as any other. Thus the location and dispersion of the points provides evidence on the coincident nature of the change in intracranial pressure. Predictability was observed at all time points (12 and 24 months). Utilizing the surrogacy analysis plots (Fig. 2), changes in headache measures can be inferred from changes in ICP (Table 3) e.g. at 12 months follow up the reduction in ICP of $-5 \mathrm{cmCSF}$ is associated with a change in headache severity of -0.95 and a mean MHD of -3.06 . Whereas a reduction in ICP of $-10 \mathrm{cmCSF}$ was associated with a reduction of headache severity of - 1.35 and mean MHD of -4.08, at 12 months (Table 3).

\section{Quality of life and intracranial pressure}

Quality of life, as measured by SF-36 PCS and MCS were 28.7 (12.7) and 37.7 (11.0) respectively at baseline and 37.7 (14.9) and 38.9 (12.2) respectively by 12 months (Table 2). There was no relationship between SF-36 scores and headache severity, MHD and ICP at baseline. 

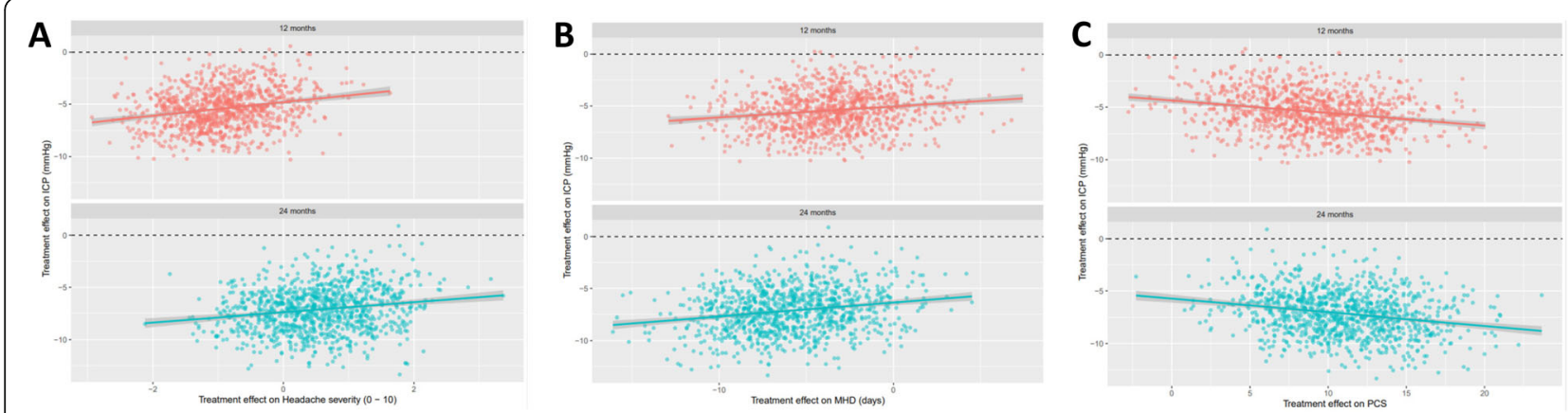

Fig. 2 Bootstrap surrogacy analysis of ICP and headache outcomes. The $x$-axis reflects change in headache outcomes with change in ICP on the $y$-axis. The lines are simple linear regressions and the shaded regions are $95 \%$ confidence intervals of the mean. Changes in intracranial pressure are plotted at 12 and 24 months. Each positive value represent improvement i.e. reduction in ICP and headaches outcomes, with the larger the angle of the slope the greater the relationship. A Illustrates headache severity on the y axis with a positive association with ICP over the time horizons. B Illustrates monthly headache days (MHD) on the y axis with a positive association with ICP over the time horizons. $\mathbf{C}$ IIlustrates quality of life physical component score (PCS) on the y axis with a positive association with ICP over the time horizons

The improvement in the PCS was associated with improving headache severity at 12 and 24 months (Fig. 3a and $\mathrm{b}, r=-0.522, p<0.001$ and $r=-0.356, p=0.04$, respectively). The PCS also improved in association with reduction in ICP at 12 months $(r=-0.546, p<0.001)$. MCS did not relate to headache or ICP. Analysis identified a positive association between ICP and PCS at 12 and 24 months (Fig. 3C).

Subanalysis by treatment assignment and disease duration was not possible due to small numbers in each group.

\section{Discussion}

Headache is a near universal sequela of IIH, and can complicate other disorders with raised ICP. We demonstrate a positive relationship between ICP and headache severity and monthly headache days, which has not been noted previously in IIH. Patients with the greatest reduction in ICP over 12 months saw the greatest reduction in headache frequency and severity, and this was associated with improvement of physical functioning in the quality of life SF-36.

We observed that the majority of patients with IIH had migraine-like headaches at baseline, as previously reported $[15,18]$. One patient was headache-free, whereas the majority described severe continuous daily headache pain associated with poor quality of life. Overall headache in patients with active IIH was of moderate pain severity, long daily duration and a mean frequency of 22 days per month, with the associated headache impacting on the individual's quality of life. These observations are in keeping with broader patients' views that the chronic daily headache of IIH is very disabling [6] and is already known to drive reduction in quality of life in IIH [8].

A previous randomized controlled trial in IIH [15] classified $68 \%$ with migraine or probable migraine and $26 \%$ tension-type or probable tension-type headaches. In the present study $90 \%$ reported migraine-like headaches and $70 \%$ would fulfil the criteria for a diagnosis of chronic migraine. Furthermore, $40 \%$ of participants in the present study described aura. In our cohort tensiontype headaches were not common, with only one patient fulfilling the IHS criteria [9]. This may reflect differences between the types of patients recruited to the two trials, for example the IIHTT [15] participants were newly diagnosed patients, whereas the IIH:WT participants reflected a more chronic disease duration (Table 1).

$68 \%$ of participants reported a prior diagnosis of migraine before being diagnosed with $\mathrm{IIH}$, of whom over half $(53 \%)$ had been diagnosed with migraine prior to the age of 18 years old and only $11 \%$ developed migraine-like headaches following the diagnosis of $\mathrm{IIH}$. This portion of patients with a prior migraine history is considerably higher than that of the general population, where for example one study found the lifetime

Table 3 Predicting changes in headache severity and monthly headache days from changes in intracranial pressure

\begin{tabular}{|c|c|c|c|}
\hline Time (months) & Change in intracranial pressure $\left(\mathrm{cmH}_{2} \mathrm{O}\right)$ & $\begin{array}{l}\text { Change in mean headache severity } \\
\text { ( } 95 \% \text { confidence interval) }\end{array}$ & $\begin{array}{l}\text { Change in mean MHD } \\
(95 \% \text { confidence interval) }\end{array}$ \\
\hline 12 & -5 & $-0.95(-2.61,0.68)$ & $-3.06(-9.61,2.91)$ \\
\hline 12 & -10 & $-1.35(-2.70,0.10)$ & $-4.08(-9.56,2.80)$ \\
\hline 24 & -5 & $0.43(-1.18,1.96)$ & $-5.32(-11.5,1.35)$ \\
\hline 24 & -10 & $-0.19(-1.94,1.61)$ & $-6.17(-13.3,0.79)$ \\
\hline
\end{tabular}


A
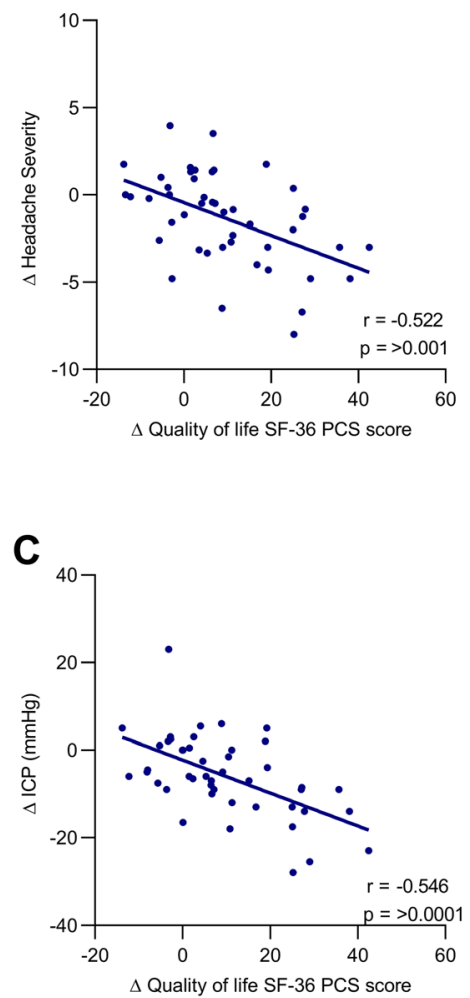

B
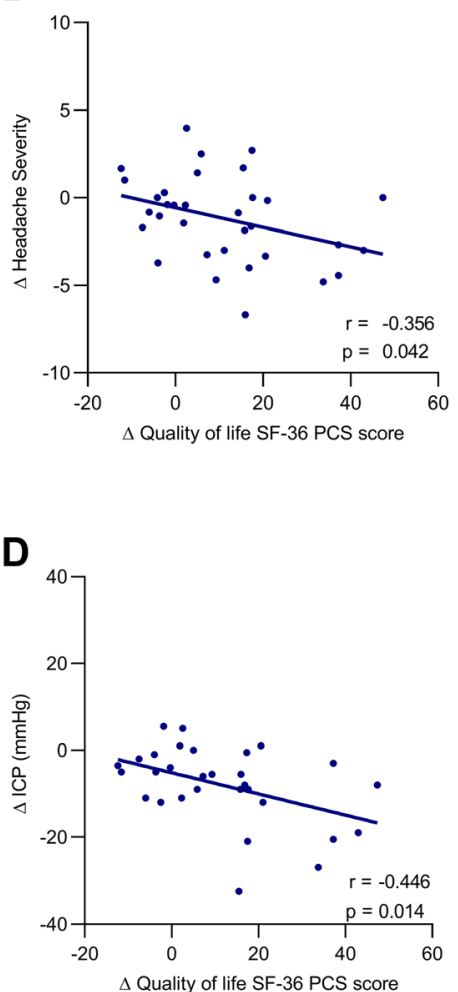

Fig. 3 Correlation of change in Quality of Life SF-36 physical component score (PCS) score and change in headache severity (HS) between baseline and 12 months (A) baseline and 24 months (B); Intracranial Pressure (ICP) between baseline and 12 months (C) between baseline and 24 months (D)

prevalence of migraine to be $29 \%$ [19]. A number of interesting observations could be postulated, for example, those with a recent diagnosis of migraine could have been initially diagnosed as migraine instead of IIH, however the portion of those with a very longstanding history of migraine are unlikely to have been misdiagnosed. Given the longevity of migraine in our cohort, some patients may have had chronic migraine before diagnosis of IIH, whereas others may have developed chronic migraine-like headaches following diagnosis. It remains unclear whether diagnosis of IIH in patients with known episodic migraine contributes towards transition to chronic migraine and if this is dependent on central sensitization.

Cutaneous allodynia is reported in over half of all patients with migraine [20]. It has not typically been a feature of conditions associated with raised ICP (e.g. brain tumours or hydrocephalus). A previous study reported allodynia in 50\% of IIH patients who mostly had a migraine-like headache profile [18]. In the present study $92 \%$ reported cutaneous allodynia at the maximum headache severity and although this was not associated with headache severity or frequency at baseline, change in allodynia over 12 months was associated with change in ICP.
Headache management is an unmet need in this disease, with no randomised controlled trials to guide treatment options. Only 18 participants were on concurrent headache preventative therapies, whereas 40 fulfilled the criteria for chronic migraine-like headaches, leaving a large portion of patients under treated. Recently the first prospective open label study of a CGRP monoclonal receptor antibody reported substantial improvements at 3 months, which continued for up to 12 months in the reduction of monthly moderate/severe headache days [13]. This benefit was seen both in those with and without prior migraine and in those with or without prior existing medication overuse headache [13]. Topiramate, a well-known migraine preventative therapy has been evaluated in IIH in the context of the impact on vision, rather than its beneficial effects of reduction of headache disability and is used off label in routine clinical practice [21]. However consensus guidelines for $\mathrm{IIH}$ placed topiramate as a useful medication for management of headaches in IIH in order to avoid medicines such as beta-blockers and tricyclics that may exacerbate weight gain, a known precipitant of the disease [1].

Similar to a previous study approximately a third of participants met the diagnostic criteria for medication- 
overuse headache at baseline [9]. Medication-overuse headache is particularly common in patients with a background of chronic migraine, for example in the United States it has been reported in up to a quarter. However, there are many factors that influence medication-overuse headache [22, 23]. Advising patients about appropriate usage of headache analgesics and avoidance of opiates is therefore an important part of headache management in IIH [1].

The data presented indicates a relationship between increased ICP and increased presence of migraine-type headache. Change in ICP can predict change in headache over time. In a previous randomized controlled trial of those with recent onset IIH (within 2 weeks of presentation) no correlation between headache characteristics and ICP was found over a 6 month follow-up [15]. Future studies are required to investigate this complex relationship from the acutely presenting patient to the more chronic phase of IIH headache. ICP monitoring studies may further delineate this complex relationship further. Therapies that have been shown to reduce ICP in animal models, such as GLP-1 [24], could be helpful in both reducing headache burden in conditions of raised ICP and weight management in IIH [25].

\section{Conclusions}

This detailed prospective evaluation of headache in patients with IIH demonstrates a consistent relationship between headache (severity and monthly headache days) and ICP. Modelling of this relationship between headache and ICP enabled prediction of headache outcomes depending on changes in ICP over a 12 and 24 month horizon. The headache in patients with active IIH is migraine-like in the majority. IIH patients demonstrated markers of allodynia which improved with reduction in ICP. Quality of life measures improved with reduction in ICP and headache. Therapeutic strategies to improve ICP are likely to improve headache in IIH.

\section{Abbreviations}

ASC-12: Allodynia Symptom Checklist-12; CGRP: Calcitonin gene-related peptide; CSF: Cerebrospinal fluid; HS: Headache severity; ICP: Intracranial pressure; $\mathrm{IH}$ : Idiopathic intracranial hypertension; $\| \mathrm{H}: \mathrm{WT}$ : $\| \mathrm{H}$ weight trial; IHS: International Headache Society; LP: Lumbar puncture; MHD: Monthly headache days; NRS: Numerical rating system; OP: Opening pressure; PCS: Physical component score

\section{Supplementary Information}

The online version contains supplementary material available at https://doi. org/10.1186/s10194-021-01321-8.

Additional file 1: Supplemental methods - allodynia checklist.

Additional file 2: Supplemental Table 1- Headache characteristics at baseline.

Additional file 3: Supplemental Table 2 - Pressure allodynia at baseline and 12 months.

\section{Acknowledgements}

No additional acknowledgements.

\section{Authors' contributions}

SM - drafting manuscript, data collection and analysis; BW - drafting manuscript and analysis; ZA, data collection and analysis; JM, data collection and analysis; $\mathrm{RO}$, data collection and analysis; $\mathrm{AY}$, data collection and analysis; MT, data collection and analysis; AG, data collection and analysis; OG, data collection and analysis; GL, data analysis and editing manuscript; AS, editing manuscript, study lead. The author(s) read and approved the final manuscript.

\section{Funding}

This study was supported by the National Institute of Health Research UK (NIHR-CS-011-028), the Medical Research Council UK (MR/K015184/1).

Availability of data and materials

Data will be made for reasonable requests.

\section{Declarations}

Ethics approval and consent to participate

The trial was approved by The National Research Ethics Committee West Midlands - The Black Country, on 28 February 2014 (14/WM/0011). All participants gave written consent after receiving detailed written

information. The trial was registered, clinicaltrials.gov identifier: NCT02124486.

\section{Consent for publication}

All co-authors consent for publication.

\section{Competing interests}

SM - Royalties - Springer publishing: Neuro-Ophthalmology, Global Trends in Diagnosis, Treatment and Management; Consultancy - Invex therapeutics, Neurodiem, Honoraria - Novartis, Santen, Santhera, Allergan, Chuagi, Chiesi; Data safety/advisory boards - Roche, Janssen, Invex therapeutics. BW - Consultancy, Invex Therapeutics; Director Ceftronics Limited; Patent pending, Uk - 1907237.0. ZA - none. JM - none. RO - none. AY - fees for educational talk - TEVA. MT - none. AG - none. OG - none. GL - none. KB - Consultancy, Invex Therapeutics; stock - Astrazenica, GlaxoSmithKline. AS - Honoraria Chiesi; Safet board/advisory - Novartis; Director / Share options - Invex therapeutics.

\section{Author details}

${ }^{1}$ Birmingham Neuro-Ophthalmology Unit, University Hospitals Birmingham, Birmingham, UK. ²University of Birmingham, Metabolic Neurology, Institute of Metabolism and Systems Research, Birmingham, UK. ${ }^{3}$ Department of Neurology, University Hospitals Birmingham, Birmingham, UK. ${ }^{4}$ University of Birmingham College of Medical and Dental Sciences, Birmingham, UK.

${ }^{5}$ Birmingham Clinical Trials Unit, University of Birmingham, Birmingham, UK.

Received: 6 May 2021 Accepted: 1 September 2021

Published online: 07 October 2021

\section{References}

1. Mollan SP, Davies B, Silver NC, Shaw S, Mallucci CL, Wakerley BR, Krishnan A, Chavda SV, Ramalingam S, Edwards J, Hemmings K, Williamson M, Burdon MA, Hassan-Smith G, Digre K, Liu GT, Jensen RH, Sinclair AJ (2018) Idiopathic intracranial hypertension: consensus guidelines on management. J Neurol Neurosurg Psychiatry 89(10):1088-1100. https://doi.org/10.1136/jnnp-201 7-317440

2. Adderley NJ, Subramanian A, Nirantharakumar K, Yiangou A, Gokhale KM, Mollan SP, Sinclair AJ (2019) Association Between Idiopathic Intracranial Hypertension and Risk of Cardiovascular Diseases in Women in the United Kingdom. JAMA Neurol 76(9):1088-1098. https://doi.org/10.1001/jama neurol.2019.1812

3. Mollan SP, Aguiar M, Evison F, Frew E, Sinclair AJ (2019) The expanding burden of idiopathic intracranial hypertension. Eye (Lond) 33(3):478-485 https://doi.org/10.1038/s41433-018-0238-5

4. Friedman DI, Liu GT, Digre KB (2013) Revised diagnostic criteria for the pseudotumor cerebri syndrome in adults and children. Neurology. 81(13): 1159-1165. https://doi.org/10.1212/WNL.0b013e3182a55f17 
5. Westgate CS, Botfield HF, Alimajstorovic Z, Yiangou A, Walsh M, Smith G, Singhal R, Mitchell JL, Grech O, Markey KA, Hebenstreit D, Tennant DA, Tomlinson JW, Mollan SP, Ludwig C, Akerman I, Lavery GG, Sinclair AJ (2021) Systemic and adipocyte transcriptional and metabolic dysregulation in idiopathic intracranial hypertension. JCI Insight 13(10):145346. https://doi. org/10.1172/jci.insight.145346. Epub ahead of print. PMID: 33848268

6. Mollan S, Hemmings K, Herd CP, Denton A, Williamson S, Sinclair AJ (2019) What are the research priorities for idiopathic intracranial hypertension? A priority setting partnership between patients and healthcare professionals. BMJ Open 9(3):e026573. https://doi.org/10.1136/bmjopen-2018-026573

7. Mollan SP, Hoffmann J, Sinclair AJ (2019) Advances in the understanding of headache in idiopathic intracranial hypertension. Curr Opin Neurol 32(1):9298. https://doi.org/10.1097/WCO.0000000000000651

8. Mulla Y, Markey KA, Woolley RL, Patel S, Mollan SP, Sinclair AJ (2015) Headache determines quality of life in idiopathic intracranial hypertension. J Headache Pain 16(1):521-521. https://doi.org/10.1186/s10194-015-0521-9

9. Headache Classification Committee of the International Headache Society (IHS) (2013) The international classification of headache disorders, 3rd edition (beta version). Cephalalgia. 33(9):629-808. https://doi.org/10.1177/ 0333102413485658

10. Yiangou A, Mitchell J, Markey KA, Scotton W, Nightingale P, Botfield H, Ottridge R, Mollan SP, Sinclair AJ (2019) Therapeutic lumbar puncture for headache in idiopathic intracranial hypertension: minimal gain, is it worth the pain? Cephalalgia. 39(2):245-253. https://doi.org/10.1177/033310241 8782192

11. Yri HM, Jensen RH (2015) Idiopathic intracranial hypertension: clinical nosography and field-testing of the ICHD diagnostic criteria. A case-control study. Cephalalgia. 35(7):553-562. https://doi.org/10.1177/03331024145501 09

12. Thaller M, Tsermoulas G, Sun R, Mollan SP, Sinclair AJ (2020:jnnn-2020325519) Negative impact of COVID-19 lockdown on papilloedema and idiopathic intracranial hypertension. J Neurol Neurosurg Psychiatry. https:// doi.org/10.1136/jnnp-2020-325519

13. Yiangou A, Mitchell JL, Fisher C, Edwards J, Vijay V, Alimajstorovic Z, Grech O, Lavery GG, Mollan SP, Sinclair AJ (2020) Erenumab for headaches in idiopathic intracranial hypertension: a prospective open-label evaluation. Headache. 61(1):157-169. https://doi.org/10.1111/head.14026

14. Yiangou A, Mitchell JL, Vijay V, Grech O, Bilton E, Lavery GG, Fisher C, Edwards J, Mollan SP, Sinclair AJ (2020 Sep 25) Calcitonin gene related peptide monoclonal antibody treats headache in patients with active idiopathic intracranial hypertension. J Headache Pain 21(1):116. https://doi. org/10.1186/s10194-020-01182-7

15. Friedman DI, Quiros PA, Subramanian PS, Mejico LJ (2017 Sep) Gao S, McDermott M, wall M; and the NORDIC IIHT study group. Headache in idiopathic intracranial hypertension: findings from the idiopathic intracranial hypertension treatment trial. Headache. 57(8):1195-1205. https://doi.org/1 $0.1111 /$ head.13153

16. Ottridge R, Mollan SP, Mitchell J et al (2017) Randomised controlled trial of bariatric surgery versus a community weight loss programme for the sustained treatment of idiopathic intracranial hypertension: the Idiopathic Intracranial Hypertension Weight Trial (IIH:WT) protocol. BMJ Open 7(9). https://doi.org/10.1136/bmjopen-2017-017426

17. Hjermstad MJ, Fayers PM, Haugen DF (2011) Studies comparing numerical rating scales, verbal rating scales, and visual analogue scales for assessment of pain intensity in adults: a systematic literature review. J Pain Symptom Manag 41(6):1073-1093. https://doi.org/10.1016/j.jpainsymman.2010.08.016

18. Ekizoglu E, Baykan B, Orhan EK, Ertas M (2012) The analysis of allodynia in patients with idiopathic intracranial hypertension. Cephalalgia 32(14):10491058. https://doi.org/10.1177/0333102412457091

19. Schramm S, Tenhagen I,Prevalence and risk factors of migraine and nonmigraine headache in older people - results of the Heinz Nixdorf recall study Schmidt B, Holle-Lee D, Naegel S, Katsarava Z, Jöckel KH, Moebus S. Cephalalgia. 2020 3:333102420977183. doi: https://doi.org/10.1177/03331 $02420977183,6,333102420977664$

20. Lipton RB, Bigal ME, Ashina S, Burstein R, Silberstein S, Reed ML, Serrano D, Stewart WF (2008) American migraine prevalence prevention advisory group. Cutaneous allodynia in the migraine population. Ann Neurol 63(2): 148-158. https://doi.org/10.1002/ana.21211

21. Celebisoy N, Gökçay F, Sirin H, Akyürekli O (2007) Treatment of idiopathic intracranial hypertension: topiramate vs acetazolamide, an open-label study.
Acta Neurol Scand 116(5):322-327. https://doi.org/10.1111/j.1600-0404.2007. 00905.x

22. Scher Al, Lipton RB, Stewart WF, Bigal M (2010) Patterns of medication use by chronic and episodic headache sufferers in the general population: results from the frequent headache epidemiology study. Cephalalgia. 30(3): 321-328. https://doi.org/10.1111/j.1468-2982.2009.01913.x

23. Kristoffersen ES, Lundqvist C (2014) Medication-overuse headache: epidemiology, diagnosis and treatment. Ther Adv Drug Saf 5(2):87-99. https://doi.org/10.1177/2042098614522683

24. Botfield HF, Uldall MS, Westgate CSJ et al (2017) A glucagon-like peptide-1 receptor agonist reduces intracranial pressure in a rat model of hydrocephalus. Sci Transl Med 9:eaan0972

25. Mollan SP, Tahrani AA, Sinclair AJ (2021) The Potentially Modifiable Risk Factor in Idiopathic Intracranial Hypertension: Body Weight. Neurol Clin Pract 11(4):e504-e507. https://doi.org/10.1212/CPJ.0000000000001063

\section{Publisher's Note}

Springer Nature remains neutral with regard to jurisdictional claims in published maps and institutional affiliations.

Ready to submit your research? Choose BMC and benefit from:

- fast, convenient online submission

- thorough peer review by experienced researchers in your field

- rapid publication on acceptance

- support for research data, including large and complex data types

- gold Open Access which fosters wider collaboration and increased citations

- maximum visibility for your research: over $100 \mathrm{M}$ website views per year

At $\mathrm{BMC}$, research is always in progress.

Learn more biomedcentral.com/submissions 